\title{
High glucose induces the proliferation of prostatic cells via downregulating MRE11
}

\author{
CHUNWEI YE ${ }^{1 *}$, YI CAI $^{2 *}$, QIAN CAI $^{1}$, SHUNHUI YUAN $^{1}$, FAN HUANG $^{1}$, XIAOFANG YANG $^{1}$, \\ SHUCHEN HE ${ }^{1}$, ZHUOHENG LI ${ }^{1}$, YANWEN WANG ${ }^{1}$, DELIN YANG ${ }^{1}$ and ZHIPENG LI ${ }^{1}$ \\ ${ }^{1}$ Department of Urology, The Second Affiliated Hospital of Kunming Medical University, Kunming, Yunnan 650101; \\ ${ }^{2}$ Department of Urology, Xiangya Hospital, Central South University, Changsha, Hunan 410008, P.R. China
}

Received July 19, 2017; Accepted January 12, 2018

DOI: $10.3892 / \mathrm{ijmm} .2018 .3549$

\begin{abstract}
The aim of the present study was to investigate the candidate genes and pathways associated with benign prostatic hyperplasia $(\mathrm{BPH})$ and diabetes. In vitro experiments were performed using normal prostatic epithelial RWPE-1 and HPr-1 cells. The cell lines were treated with a high-glucose solution and MTS and bromodeoxyuridine assays were used to assess cell viability. Transcriptome sequencing was used to screen the candidate genes. The expression of candidate genes was further verified by reverse transcription-quantitative polymerase chain reaction (RT-qPCR) and western blotting. A meiotic recombination 11 (MRE11) overexpression vector was designed and transfected into RWPE-1 cells to verify the function of MRE11. A streptozotocin-induced diabetic rat model was established and rat MRE11 levels were determined by RT-qPCR and immunohistochemical staining. High concentrations of glucose resulted in RWPE-1 and HPr-1 cells with high viability. Transcriptome sequencing revealed that MRE11 was downregulated when RWPE-1 cells were exposed to high-glucose conditions. When MRE11 was overexpressed, cell viability decreased and cell apoptosis was induced under high-glucose conditions. Prostatic tissues from rats were collected and assessed; MRE11 expression was observed to be decreased, which was consistent with the in vitro cell experiments. BPH may be associated with diabetes, as MRE11 expression in prostatic cells was decreased when exposed to high-glucose conditions. Therefore, MRE11 may have potential as a biomarker for the early diagnosis of BPH and diabetes.
\end{abstract}

Correspondence to: Dr Delin Yang or Dr Zhipeng Li, Department of Urology, The Second Affiliated Hospital of Kunming Medical University, 374 Dianmian Road, Kunming, Yunnan 650101, P.R. China

E-mail: 444307491@qq.com

E-mail: qh2518@163.com

${ }^{*}$ Co-first authors

Key words: benign prostatic hyperplasia, diabetes, high glucose, meiotic recombination 11

\section{Introduction}

Benign prostatic hypertrophy (BPH) is the most common benign tumor in males, characterized by an increased number of glandular and connective cells in the prostate and (1). BPH typically causes prostate enlargement and the secondary condition of chronic bladder outlet obstruction (2). In addition, urinary retention, gross hematuria, renal insufficiency and bladder cancer may arise. BPH may also lead to prostate cancer, which is the leading cause mortality due to cancer in males $(3,4)$. A previous study demonstrated that the pathogenesis of BPH is multifactorial, with factors including age, diabetes, obesity and ethnicity influencing its development (5). Reports on the association between diabetes and BPH are conflicting; some have suggested that there is a positive association (6), whereas others have reported no association (7).

Type 2 diabetes mellitus (T2D), characterized by persistent hyperglycemia, affects $>370$ million people worldwide and is a major cause of morbidity and mortality (8). T2D is a disease in which tissues are resistant to the effects of insulin and/or insulin production is insufficient to meet requirements (1). The consequence of this is an inability to regulate blood sugar, resulting in chronic hyperglycemia. For many decades, therapeutic strategies aimed at decreasing glucose levels have focused on agents that stimulate the release of insulin from the pancreas or improve insulin-stimulated glucose uptake in peripheral tissues (9).

Little data is available regarding the association between diabetes and BPH; however, statistical analysis suggests that there is an overlap in males who suffer from BPH and diabetes, indicating that there may be an existing association between these diseases (10-12). Human genetic studies allow for the investigation of novel molecules and pathways that contribute to natural variation in specific traits, including blood glucose levels or the risk of disease. According to numerous studies, the number of genes involve in the pathogenic processes of T2D have been reported. For example, phosphoinositide 3-kinase/protein kinase B signaling activates forkhead box Os (FoxOs), which protects against oxidative stress to prevent the death of $\beta$ pancreatic cells (13-15). FoxO1 has been reported to control various cellular processes, including the stress response, proliferation and differentiation $(16,17)$. As nutrient and FoxO factors are closely associated, FoxO may also serve an important role 
in cell-secreted hormones that regulate glucose homeostasis. These genes are important for regulating glucose homeostasis and may therefore be associated with diabetes and potentially $\mathrm{BPH}$. However, few findings have validated the genes involved in diabetes and BPH. In our previous study (18), a cDNA microarray analysis was performed to identify the effects of diabetes on gene expression profiles in prostate samples. A cell culture model (human normal prostatic RWPE-1 cell line) was also employed to study the direct effects of high glucose (14). The results indicated that high glucose was associated with the downregulation of antioxidative enzymes and DNA damage repair genes, including MRE11.

In the present study, an in vitro experiment was conducted using prostatic cell lines RWPE-1 and HPr-1 to verify the effects of high-glucose solution. Transcriptome sequencing was subsequently performed to screen the candidate genes that may be involved in the pathogenic process of prostatic enlargement. Following validation of the candidate genes by transfection of an overexpression vector, an in vivo experiment was performed using rats with streptozotocin (STZ)-induced $\mathrm{T} 2 \mathrm{D}$ to further invetsigate the role of these genes in diabetes.

\section{Materials and methods}

Cell culture. Normal prostatic epithelial RWPE-1 and HPr-1 cells (Institute of Biochemistry and Cell Biology, Chinese Academy of Sciences, Shanghai, China) were cultured in complete keratinocyte serum-free medium (Sigma-Aldrich; Merck KGaA, Darmstadt, Germany), supplemented with $1 \%$ penicillin/streptomycin/amphotericin B, $50 \mu \mathrm{g} / \mathrm{ml}$ bovine pituitary extract (Absin Bioscience, Inc., Shanghai, China) and $5 \mathrm{ng} / \mathrm{ml}$ epidermal growth factor (Thermo Fisher Scientific, Inc., Waltham, MA, USA). The cells were incubated at $37^{\circ} \mathrm{C}$ in an atmosphere containing $5 \% \mathrm{CO}_{2}$. When cells reached $70-80 \%$ confluence they were washed with PBS and detached using $0.25 \%$ trypsin/ $0.2 \%$ EDTA. Cells were viewed under a light microscope (magnification, x200; Eclipse T1, Nikon Corporation, Tokyo, Japan), and subsequently suspended at a concentration of $1 \times 10^{6}$ cells $/ \mathrm{ml}$.

MTS and bromodeoxyuridine (BrdU) proliferation activity assays. Cell proliferation was analyzed via an MTS assay. Cells were seeded at a density of $4 \times 10^{3}$ cells/well in 96-well plates. The high-glucose solution was diluted with culture medium at 0 , 5.5, 25, 50 and $75 \mathrm{mM}$, which was added to each well and incubated for $48 \mathrm{~h}$ at $37^{\circ} \mathrm{C}$. Following determination of the optimal concentration, cells were incubated with $50 \mathrm{mM}$ high glucose solution (Sigma-Aldrich; Merck KGaA) for 0, 24, 48 and $72 \mathrm{~h}$.

For the MTS assay, $20 \mu \mathrm{l}$ MTS reagent (Promega Corp., Madison, WI, USA) was added to the cells and incubated for $3 \mathrm{~h}$ at $37^{\circ} \mathrm{C}$ in an atmosphere containing $5 \% \mathrm{CO}_{2}$. The plates were then read at $490 \mathrm{~nm}$ with a microplate reader (Thermo Fisher Scientific, Inc.). Each sample was analyzed in triplicate.

A BrdU Cell Proliferation Assay kit was used according to the manufacturer's protocols (BioVision, Inc., Milpitas, CA, USA). Briefly, 10X BrdU solution was added to the wells and incubated for $3 \mathrm{~h}$ at $37^{\circ} \mathrm{C}$ in an atmosphere containing $5 \% \mathrm{CO}_{2}$. The culture medium was removed and $100 \mu \mathrm{l}$ fixing/denaturing solution was added to each well at room temperature for $30 \mathrm{~min}$. The supernatant was removed and $100 \mu \mathrm{l} 1 \mathrm{X} \mathrm{BrdU}$ detection antibody solution was added. Cells were incubated at room temperature for a further $1 \mathrm{~h}$. Prior to washing with $1 \mathrm{X}$ wash buffer twice, the supernatant was removed. A total of $100 \mu \mathrm{l} 1 \mathrm{X}$ anti-mouse horseradish-peroxidase-linked antibody solution (provided in the kit) was added to each well and incubated at room temperature for $1 \mathrm{~h}$. The supernatant was removed and cells were washed three times with wash buffer. 3,3',5,5'-tetramethylbenzidine substrate $(100 \mu 1)$ was added into each well and absorbance was measured at $650 \mathrm{~nm}$ for 5-30 min. To terminate color development, $100 \mu \mathrm{l}$ stop solution was added into each well and the absorbance at $450 \mathrm{~nm}$ was measured.

Apoptosis and cell cycle assay. Flow cytometric analysis was performed to assess apoptosis and cell cycle distribution in RWPE- 1 and HPr-1 cells. The cells were seeded at a density of $1 \times 10^{6}$ cells/well in 6-well plates and allowed to attach for $48 \mathrm{~h}$ at $37^{\circ} \mathrm{C}$ prior to the onset of treatments. Following treatment, cells were detached using EDTA-free trypsin for $5 \mathrm{~min}$ at room temperature. Apoptosis was assessed using an Annexin V assay kit (FITC Annexin V Apoptosis Detection Kit with 7-AAD, BD Biosciences, Franklin Lakes, NJ, USA) according to manufacturer's protocols. For the cell cycle assay, cells were centrifuged at $500 \mathrm{x} \mathrm{g}$ for $10 \mathrm{~min}$ at $4^{\circ} \mathrm{C}$ and subsequently incubated in $500 \mu \mathrm{l}$ hypotonic DNA staining buffer $(1 \mathrm{~g} / 1$ sodium citrate, $0.3 \%$ Triton-X 100, $0.1 \mathrm{~g} / 1$ propidium iodide, $0.02 \mathrm{~g} / 1$ Ribonuclease A in distilled water; BD Biosciences) for $30 \mathrm{~min}$ at $4^{\circ} \mathrm{C}$. Analyses were performed with a BD Accuri C6 Flow Cytometer (BD Biosciences). Cell cycle data were analyzed with FlowJo software v 7.6 (Tree Star, Inc., Ashland, OR, USA).

Transcriptome sequencing. RNA was extracted from cells using TRIzol (Invitrogen; Thermo Fisher, Scientific, Inc.) following the manufacturer's protocols. RNA was quantified using a Qubit RNA assay (Thermo Fisher Scientific, Inc.) and the quality was evaluated by Agilent bioanalyzer 2100 (Agilent Technologies, Inc., Santa Clara, CA, USA). A total of $\sim 1,000$ ng RNA was used for library preparation with the VAHTST mRNA-seq v2 Library Prep kit (Illumina, Inc., San Diego, CA, USA). Briefly, RNA with a polyA tail was captured using Dynaloligo beads (Invitrogen; Thermo Fisher Scientific, Inc.). The RNA was fragmented and reverse transcribed into double-strand cDNA using SuperScript III cDNA Synthesis kit at $70^{\circ} \mathrm{C}$ for $5 \mathrm{~min}$ (Thermo Fisher Scientific, Inc.). Thermocycling conditions were as follows: 30 cycles of $94^{\circ} \mathrm{C}$ for $15-30 \mathrm{sec}, 55-65^{\circ} \mathrm{C}$ for $30 \mathrm{sec}$ and $72^{\circ} \mathrm{C}$ for $1 \mathrm{~min}$. The ends of the cDNA were repaired by the End-It DNA EndRepair kit (End-It DNA END Repair kit; Epicentre; Illumina, Inc.) and treated via A-addition. The modified cDNA was ligated to adapters and subjected to quantitative polymerase chain reaction (qPCR) amplification (described later). The library quality was determined using the Agilent bioanalyzer 2100. The libraries were sequenced with an Illumina HiSeq 4000 system. Reads were aligned to the reference genome (GRCH37/hg19) by TOPHAT v.2.1.0 (19). The reads per kilobase of exon model per million mapped reads of each gene were calculated. The differential expression signals were calculated and P-values were adjusted using Benjamini and Hochberg's approach (20). Enrichment of the differentially expressed genes in Kyoto 
Encyclopedia of Genes and Genomes pathway was tested using KOBAS 2.0 software (KOBAS, London, UK).

Lentivirus transfection. The full length of MRE11 cDNA was cloned into pCDNA3.1 vector (Thermo Fisher Scientific, Inc.). $293 \mathrm{~T}$ cells (Thermo Fisher Scientific, Inc.) were seeded in a $10 \mathrm{~cm}$ plate and transfected with the pCDNA3.1 vector using RNAi-Mate transfection reagent (Thermo Fisher Scientific, Inc.). Cells were cultured at $37^{\circ} \mathrm{C}$ until they covered $80-90 \%$ of the plate, at which point they were then harvested with trypsin and amplified in $15 \mathrm{~cm}$ plate at $37^{\circ} \mathrm{C}$ in an atmosphere containing $5 \% \mathrm{CO}_{2}$ overnight. The pCDNA3.1-MRE11 vector and package vectors (pGag/Pol, pRev and pVSV-G; GenePharm, Inc., Sunnyvale, CA, USA) were mixed in $1.5 \mathrm{ml}$ Dulbecco's modified Eagle's medium (DMEM) without serum (GenePharm, Inc.) and incubated at room temperature for 5 min. A total of $300 \mu \mathrm{l}$ of RNAi-Mate (GenePharm, Inc.) was prepared and diluted in $1.5 \mathrm{ml}$ DMEM, which was then mixed with the vectors mixture and incubated for 20-25 min at room temperature to form the transfection complex. The cell culture medium was removed and replaced with $8 \mathrm{ml}$ DMEM. The transfection complex was added to the cells and incubated for $4-6 \mathrm{~h}$ at $37^{\circ} \mathrm{C}$ in an atmosphere containing $5 \% \mathrm{CO}_{2}$. The culture medium was removed and replaced with RPMI-1640 medium with serum (Thermo Fisher Scientific, Inc.), and incubated for $72 \mathrm{~h}$ at $37^{\circ} \mathrm{C}$ in an atmosphere containing $5 \% \mathrm{CO}_{2}$. The cell culture supernatant was collected and centrifuged at $1,788 \mathrm{x} \mathrm{g}$ for $4 \mathrm{~min}$ at $4^{\circ} \mathrm{C}$. The supernatant was filtered through a $0.45 \mu \mathrm{m}$ filter and subsequently centrifuged at $4,472 \mathrm{x} g$ for $2 \mathrm{~h}$ at $4^{\circ} \mathrm{C}$. The viral solution was stored at $-80^{\circ} \mathrm{C}$ prior to use. $293 \mathrm{~T}$ cells were seeded in 96-well plates at a density of $3 \times 10^{4}$ cells/well. The viral solution was diluted 10x with RPMI-1640 medium to give five different concentrations and the final titer was $2 \times 10^{8} \mathrm{TU} / \mathrm{ml}$. The culture medium was removed from each well and supplemented with $100 \mu \mathrm{l}$ of the viral solution. Normal saline was used as control. Cells were incubated at $37^{\circ} \mathrm{C}$ in an atmosphere containing $5 \% \mathrm{CO}_{2}$ for $24 \mathrm{~h}$. The cell culture supernatant was removed and replaced with $100 \mu 1$ RPMI-1640 medium. Cells were incubated for $72 \mathrm{~h}$ at $37^{\circ} \mathrm{C}$. Fluorescence microscopy (magnification, x200) was used to detect fluorescence and the titer of virus was calculated according to the dilution ratio. RWPE-1 cells were then transfected with the lentivirus to generate MRE11-overexpressing cells when the cell density reached $60-70 \%$.

Animal experiment. All animal experiments were approved by the Institutional Animal Care and Use Committee of Kunming Medical University. A total of 20 male Sprague Dawley rats (12 weeks old; weight, 230-280 g) were obtained from Sun Yay-sen University Experimental Animal Center (Guangzhou, China). The rats were maintained at a constant temperature of $24^{\circ} \mathrm{C}, 45-55 \%$ humidity with a $12 \mathrm{~h}$ light/dark cycle and were fed standard food pellets with free access to sterile water. Rats were randomly divided into two groups $(n=6-12)$. Diabetes was induced by intraperitoneal injection with STZ $(60 \mathrm{mg} / \mathrm{kg}$, Sigma Aldrich; Merck KGaA). Blood glucose levels were monitored by harvesting blood samples at weeks 2 and 4 to confirm the presence of diabetes. The rats were sacrificed by an intraperitoneal injection of pentobarbital $(160 \mathrm{mg} / \mathrm{kg}$, Sigma Aldrich; Merck $\mathrm{KGaA}$ ) at 4 weeks following the induction of diabetes.
Prostate tissues were harvested and stored at $-80^{\circ} \mathrm{C}$ for $\mathrm{qPCR}$ assay or fixed in formalin for 24-48 $\mathrm{h}$ at room temperature and sectioned $(2.5 \mu \mathrm{m})$ for immunohistochemical staining.

Immunohistochemical staining. The formalin-fixed paraffin-embedded tissues were deparaffinized and washed twice with PBS. The tissue was blocked with $3 \% \mathrm{H}_{2} \mathrm{O}_{2}$ for 5-20 min at room temperature and subsequently washed three times with PBS. Tissues were fixed via water incubation at $95^{\circ} \mathrm{C}$ for $10 \mathrm{~min}$ and washed with PBS. The tissue was then blocked with 5\% BSA for $10 \mathrm{~min}$ at room temperature (Sigma Aldrich; Merck KGaA) and incubated with primary antibodies (Anti-Mre11, ab109623, 1:1,000, Abcam, Cambridge, MA, USA; anti-PCNA, sc-25280, 1:500, Santa Cruz Biotechnology, Inc., Dallas, TX, USA) at $4^{\circ} \mathrm{C}$ overnight. Following three washes with PBS, the tissue was incubated with a horseradish peroxidase-conjugated secondary antibody secondary antibody (SV0002; 1:1,000; Wuhan Boster Biological Technology, Ltd., Wuhan, China) for $1 \mathrm{~h}$ at room temperature. Following washing with PBS, the sections were developed in freshly prepared 3,3'-diaminobenzidine solution for $30 \mathrm{sec}$ at room temperature and then counterstained with hematoxylin for $45 \mathrm{sec}$ at room temperature, rehydrated with a graded series of ethanol, washed with xylene and mounted at room temperature. The expression of MRE-11 and PCNA was estimated by light microscopy (magnification, x200).

Reverse transcription (RT)-qPCR assay. Total RNA from sampled cells and tissues was extracted using TRIzol according to the manufacturer's protocols. Genomic DNA-free RNA was then converted into cDNA using the M-MLV Reverse Transcriptase at $42^{\circ} \mathrm{C}$ for $60 \mathrm{~min}$ (Promega Corp.). qPCR was performed using SYBR Green PCR Master Mix (Bio-Rad Laboratories, Inc., Hercules, CA, USA). $\beta$-actin was used as the reference gene. Primers sequences were as follows: MRE11, forward 5'-AACGGGAACGTCTGGGTA AT-3' and reverse 5'-GGCTAAAGCGAAGAACACTGAA-3'; $\beta$-actin, forward 5'-CATGTACGTTGCTATCCAGGC-3' and reverse 5'-CTCCTTAATGTCACGCACGAT-3'. The thermo cycling conditions were as follows: $95^{\circ} \mathrm{C}$ for $2 \mathrm{~min}$ followed by 30 cycles of $95^{\circ} \mathrm{C}$ for $30 \mathrm{sec}, 60^{\circ} \mathrm{C}$ for $30 \mathrm{sec}$ and $72^{\circ} \mathrm{C}$ for $1 \mathrm{~min}$ and $72^{\circ} \mathrm{C}$ for $1 \mathrm{~min}$ with a final extension step at $72^{\circ} \mathrm{C}$ for $10 \mathrm{~min}$. Fold changes in expression were calculated using the $2^{-\Delta \Delta C q}$ method (21) using iCycler iQ software v.3.0 (Bio-Rad Laboratories, Inc.).

Western blot analysis. Protein was extracted from cells and tissues and the concentration was determined using a BCATM-Protein Assay kit (Guangzhou Fulengen, Co., Ltd., Guangzhou, China). A total of $10 \mu \mathrm{l}$ protein was subjected to $10 \%$ SDS-PAGE. Protein was then transferred onto nitrocellulose membranes, which was blocked with $5 \%$ non-fat milk in phosphate-buffered saline containing $0.1 \%$ Tween-20 for $1 \mathrm{~h}$ at room temperature. Subsequently, the membranes were incubated overnight at $4{ }^{\circ} \mathrm{C}$ with the following primary antibodies: Anti-Mre11, anti-phosphorylated checkpoint kinase 2 (p-CHK2; CST Biological Reagents Co., Ltd., Shanghai, China), anti-p-M-phase inducer phosphatase 1 (CDC25A; ab75743; Abcam), anti-CDC25A (BA3149; Wuhan Boster Biological Technology) anti-p-cyclin dependent kinase (CDK2; 2561; CST 

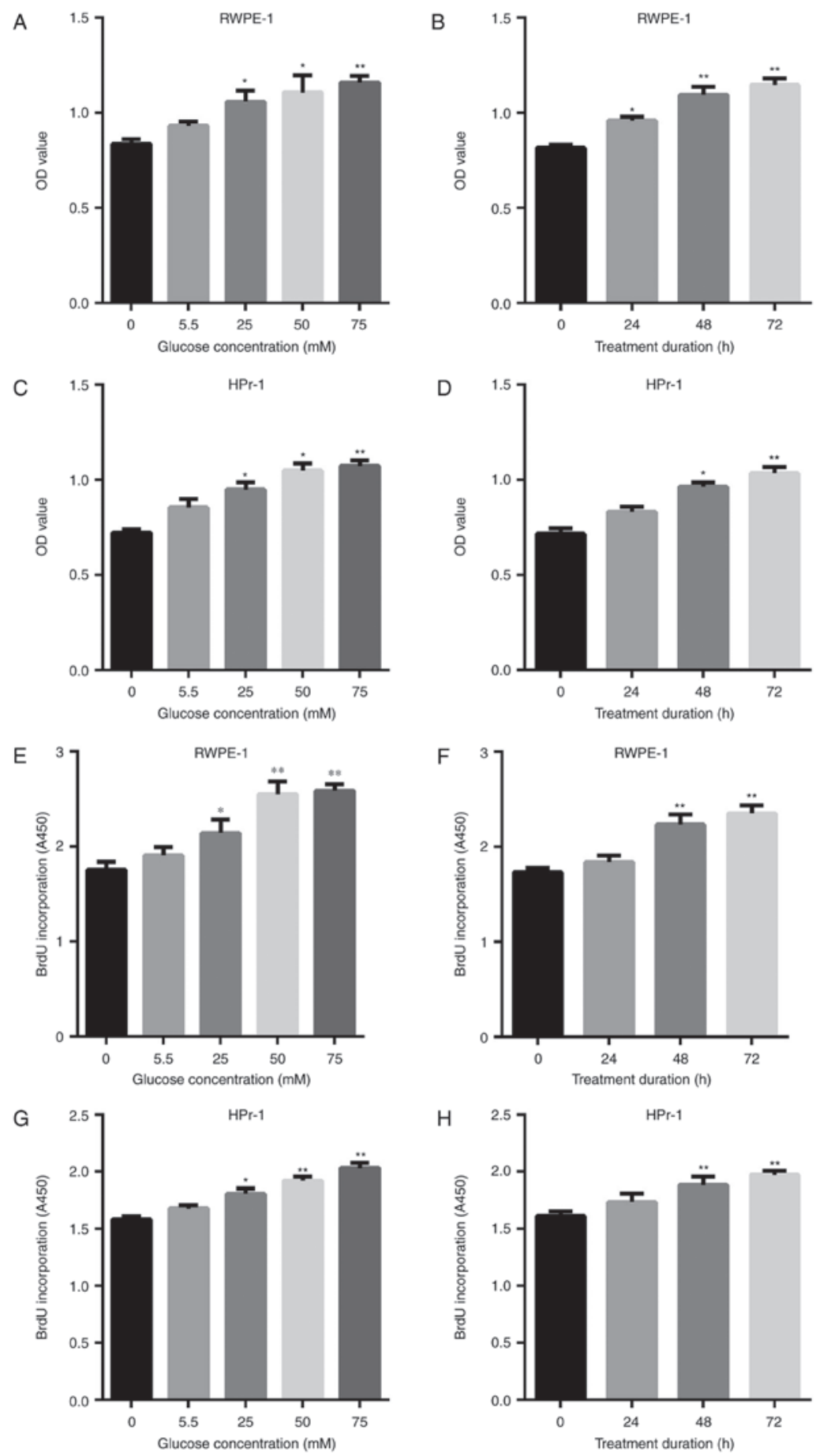

Figure 1. Cell viability was assessed using MTS and BrdU assays to determine the optimal concentration and duration of treatment with high glucose solution. (A) RWPE-1 cells were treated with 0, 5.5, 25, 50 or $75 \mathrm{mM}$ glucose for $48 \mathrm{~h}$ or (B) for $0,24,48$ or $72 \mathrm{~h}$ with $50 \mathrm{mM}$ glucose and assessed using an MTS assay. (C) HPr-1 cells were treated with $0,5.5,25,50$ or $75 \mathrm{mM}$ glucose for $48 \mathrm{~h}$ or (D) for $0,24,48$ or $72 \mathrm{~h}$ with $50 \mathrm{mM}$ glucose and assessed using an MTS assay. (E) RWPE-1 cells were treated with $0,5.5,25,50$ or $75 \mathrm{mM}$ glucose for $48 \mathrm{~h}$ or (F) for $0,24,48$ or $72 \mathrm{~h}$ with $50 \mathrm{mM}$ glucose and assessed using a BrdU assay. (G) HPr-1 cells were treated with $0,5.5,25,50$ or $75 \mathrm{mM}$ glucose for $48 \mathrm{~h}$ or $(\mathrm{H})$ for $0,24,48$ or $72 \mathrm{~h}$ with $50 \mathrm{mM}$ glucose and assessed using a BrdU assay. ${ }^{*} \mathrm{P}<0.05$ and ${ }^{* *} \mathrm{P}<0.01$ vs. control $(0 \mathrm{mM}$ or $0 \mathrm{~h})$. BrdU, bromodeoxyuridine; OD, optical density.

Biological Reagents Co., Ltd.), anti-CDK2 (ab32147; Abcam) and anti-GAPDH (HC301; TransGen Biotech, Inc., Beijing, China). All primary antibodies were used at a dilution of 1:1,000. Following three washes with TBST, membranes were incubated with a horseradish peroxidase-conjugated secondary antibody (1:2,000; ab6789; Abcam) for $2 \mathrm{~h}$ at room temperature. GAPDH was used as the loading control. The complexes were detected by enhanced chemiluminescence (Forevergen,
Guangzhou, China). ImageJ v.1.6.0 (National Institutes of Health, Bethesda, MD, USA) was used for densitometry.

Statistical analysis. All data are presented as the mean \pm standard deviation and at least three replicates of each assay were performed. Normally distributed data were compared using independent t-tests, one-way analysis of variance (ANOVA) or two-way ANOVA and Student Newman 
A
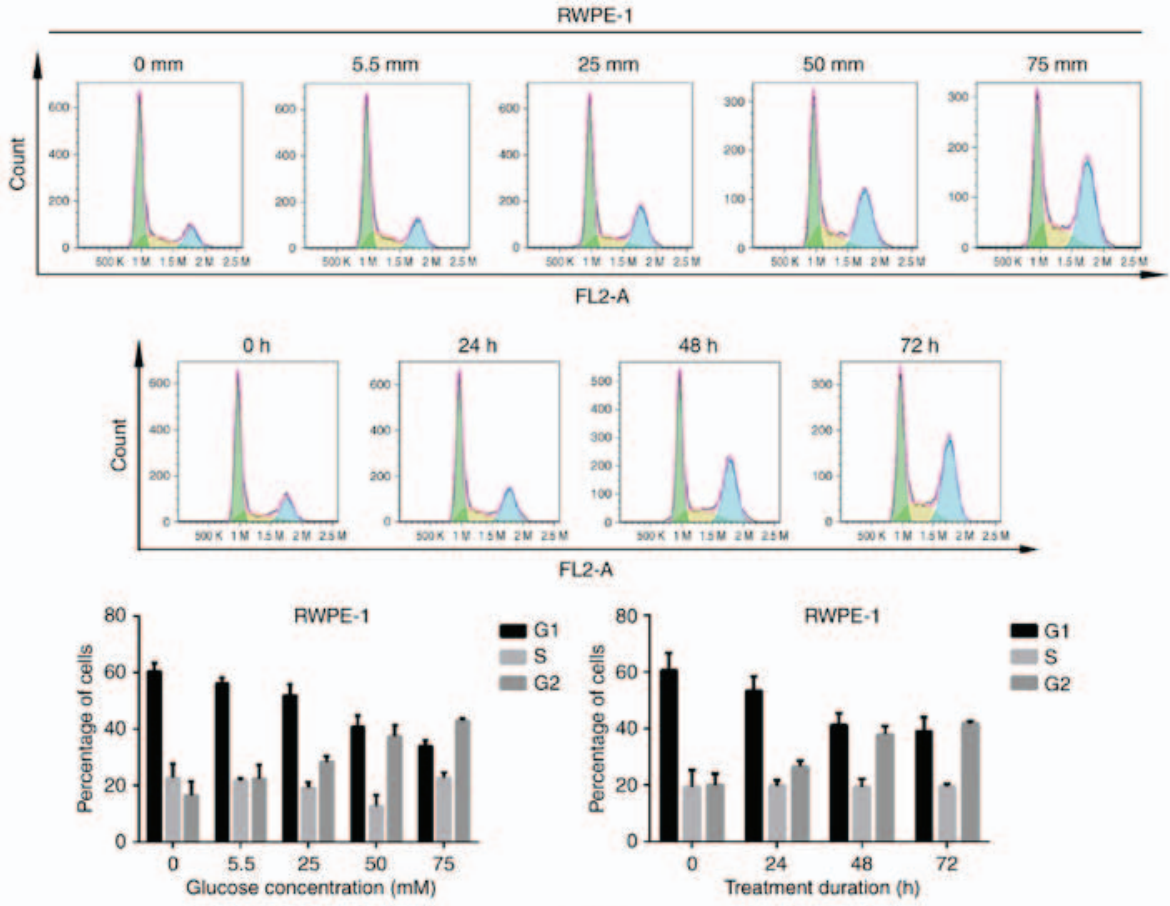

B
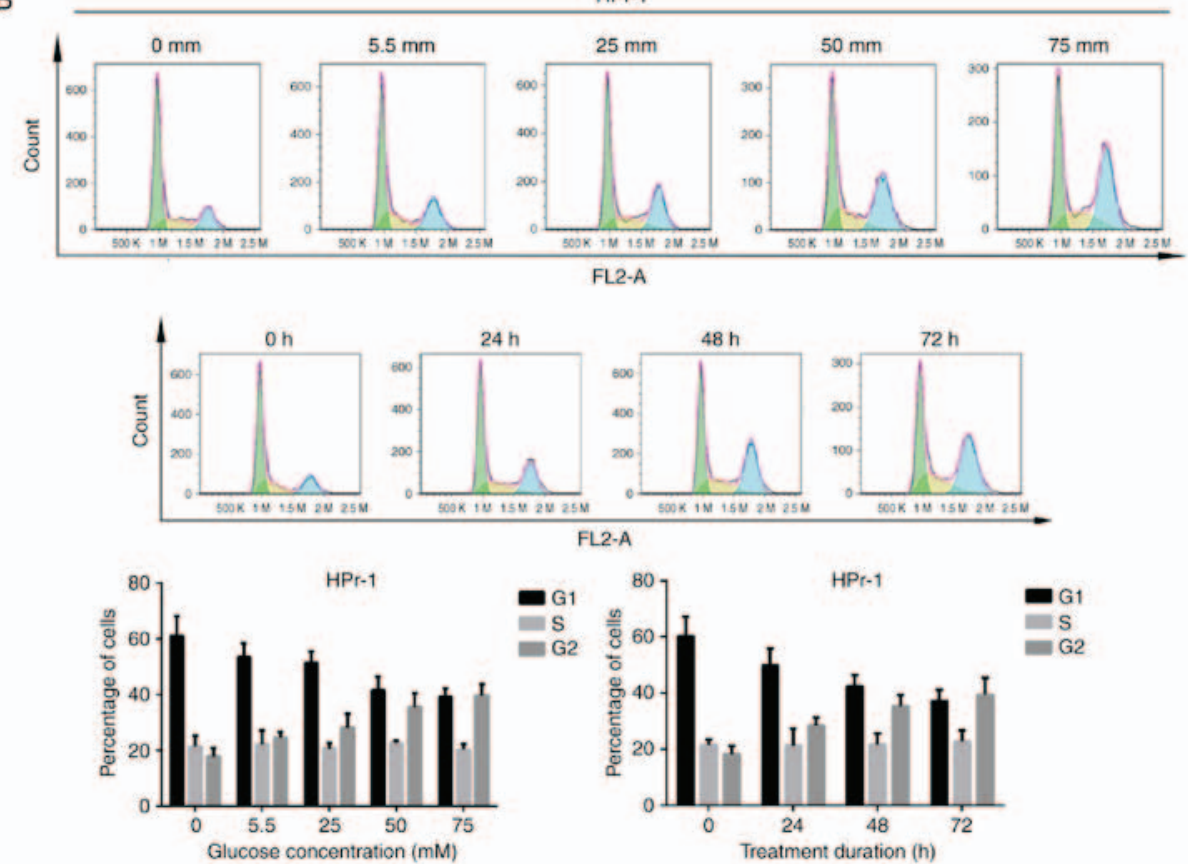

Figure 2. Cell cycle distribution of (A) RWPE-1 and (B) HPr-1 cells treated with 0, 5.5, 25, 50 or $75 \mathrm{mM}$ glucose for 0, 24, 48 and $72 \mathrm{~h}$ was assessed using flow cytometry.

Keul's post hoc test. For abnormally distributed data, the non-parametric Mann-Whitney U-test, Kolmogorov-Smirnov test, Kruskal-Wallis test and Wilcoxon test were performed. $\mathrm{P}<0.05$ was considered to indicate a statistically significant difference. SPSS 18.0 (SPSS, Inc., Chicago, IL, USA) and GraphPad Prism software 6.0 (GraphPad Software, Inc., La Jolla, CA, USA) were used for statistical analysis.

\section{Results}

High glucose concentration induces the proliferation of RWPE-1 and HPr-1 cells. Cell viability was determined using
MTS and BrdU methods (Fig. 1). Incubation in high glucose medium significantly induced the proliferation of RWPE-1 (Fig. 1A-D) and HPr-1 (Fig. 1E-H) cells in a dose-dependent manner. A concentration of $50 \mathrm{mM}$ of glucose was determined to be the optimal concentration, as treatment with $75 \mathrm{mM}$ did not markedly increase cell viability.

To further assess the effect of high glucose, flow cytometry was performed (Fig. 2). Incubation with high glucose solution markedly increased the number of cells at G2 phase and decreased the cell number at G1 phase. No significant differences were observed in the number of the cells at $S$ phase. For both cell lines, the effect was significantly induced with 

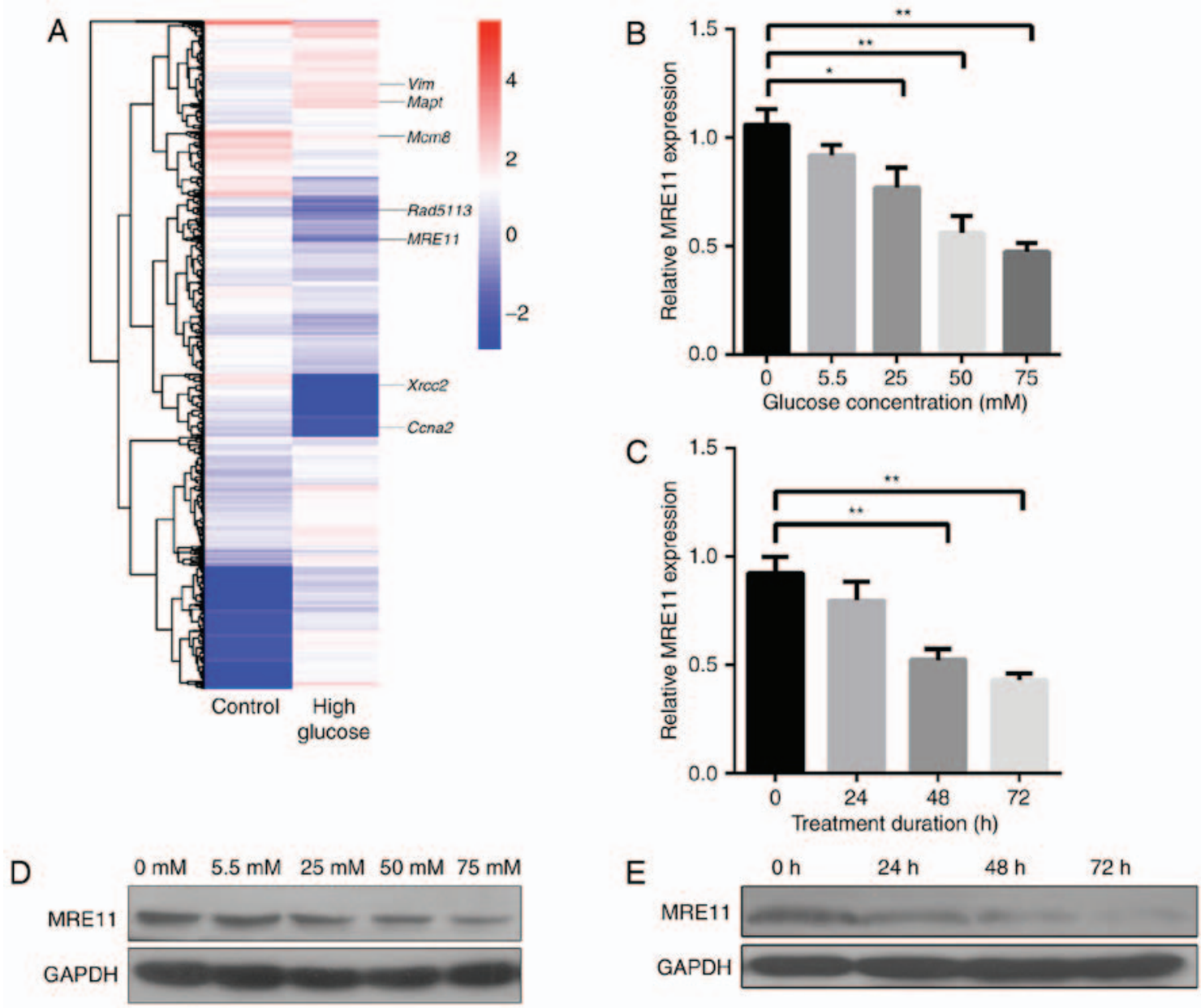

Figure 3. MRE11 was downregulated under high glucose conditions. (A) Hierarchical cluster results of transcriptome sequencing. The high glucose group was treated with $50 \mathrm{mM}$ glucose solution for $48 \mathrm{~h}$ and the control group was treated with saline. MRE11 mRNA expression of cells treated with (B) varying concentrations of glucose or for $(\mathrm{C})$ varying durations was assessed by reverse transcription-quantitative polymerase chain reaction. MRE11 protein expression of cells treated with (D) varying concentrations of glucose or for (E) varying durations was assessed by western blotting. Vim, Vimentin; Mapt, microtubule-associated protein tau; Mcm8, minichromosome maintenance complex component 8; Rad511, RAD51-like 3; MRE11, meiotic recombination 11; Xrcc2, $\mathrm{X}$-ray repair cross-complementing protein 2 ; Ccna2, cyclin a2. ${ }^{*} \mathrm{P}<0.05$ and ${ }^{* * *} \mathrm{P}<0.01$.

$50 \mathrm{mM}$ glucose solution and higher concentrations did not markedly increase the number of cells at G2 phase. Therefore, $50 \mathrm{mM}$ of glucose solution may be the optimal concentration to enhance cell proliferation. In the present study, $50 \mathrm{mM}$ glucose solution was used to verify the optimal duration of treatment. The results of flow cytometry analysis demonstrated that cell number peaked at $48 \mathrm{~h}$ and did not markedly increase with $72 \mathrm{~h}$ treatment.

MRE11 is downregulated under high glucose conditions. To verify the underlying mechanism of high-glucose induced cell proliferation, transcriptome sequencing was performed (Fig. 3). Experimental RWPE-1 cells were treated with $50 \mathrm{mM}$ glucose solution for $48 \mathrm{~h}$ and control cells were treated with saline. A hierarchical cluster graph was generated for the two groups of cells (Fig. 3A). According to the results, MRE11 was significantly downregulated in the experimental group, indicating that high concentration glucose solution may enhance cell proliferation by downregulating MRE11.

To further assess MRE11 expression, RT-qPCR and western blotting were performed. The results were consistent with those of transcriptome sequencing. As presented in Fig. 3B, the expression of MRE11 mRNA was decreased in response to treatment with high-glucose solution. At $50 \mathrm{mM}$, the mRNA expression levels of MRE11 were reduced by $\sim 50 \%$ compared with the control group (Fig. 3B). Similarly, the expression of MRE11 decreased to $\sim 50 \%$ in response to treatment with $50 \mathrm{mM}$ glucose solution for $48 \mathrm{~h}$ (Fig. 3C). Protein expression followed a similar trend to mRNA (Fig. 3D and E). These results suggest that MRE11 expression is downregulated by high glucose treatment.

MRE11 induces cell apoptosis following high glucose treatment. The role of MRE11 in proliferation regulation remains to be elucidated. In the present study, RWPE-1 cells underwent transfection to overexpress MRE11. The vector was successfully transfected and cells expressed $\sim 10$-fold move MRE11 compared cells transfected with at empty vector (Fig. 4A and B). Cells were further analyzed to detect the expression of genes of the MRE11 regulation network (Fig. 4C). When high glucose treatment was performed, the expression of p-CHK2 was markedly downregulated in normal RWPE-1 cells; however, no significant difference was observed in the MRE11-overexpressing cells regardless of glucose treatment. 

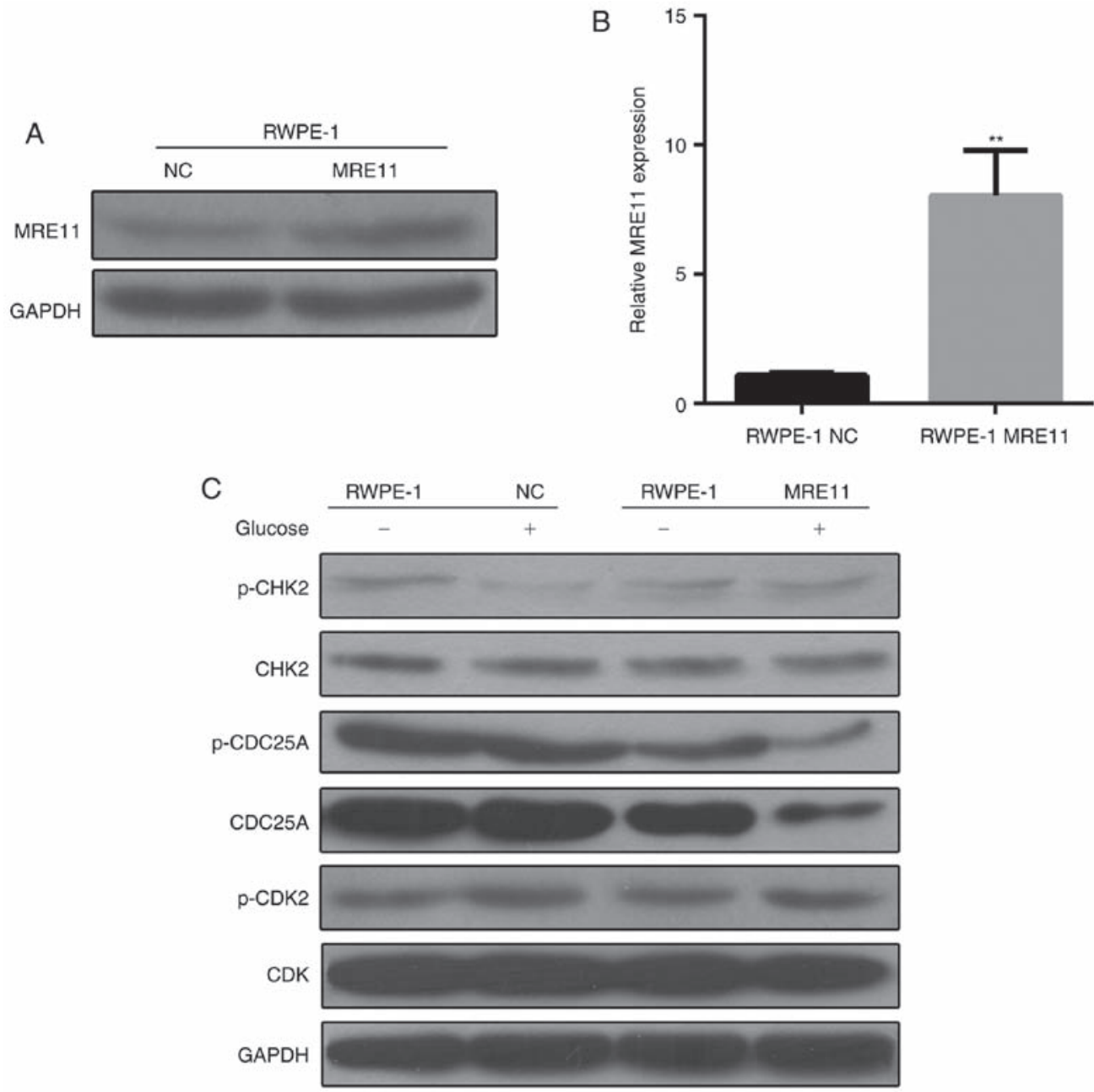

Figure 4. MRE11 overexpression. Protein and mRNA expression levels of MRE11 were determined by (A) western blotting and (B) reverse transcription-quantitative polymerase chain reaction in RWPE-1 cells following transfection with NC or MRE11-overexpression vectors. (C) Protein expression levels of the MRE11 regulation network-associated genes in RWPE-1 cells transfected with NC and MRE11-overexpression vectors. GAPDH was used as internal control. ${ }^{* *} \mathrm{P}<0.01$ vs. RWPE-1 NC. MRE11, meiotic recombination 11; NC, negative control; p, phosphorylated; CHK2, checkpoint kinase 2; CDC25A, p-M-phase inducer phosphatase 1 ; CDK, cyclin-dependent kinase; $\mathrm{H}$, high glucose.

These results suggest that the expression of p-CHK2 is dependent on MRE11 levels, as high glucose was demonstrated to downregulate MRE11. However, CDC25A expression was downregulated when MRE11 was overexpressed and high glucose treatment was applied. The results of the present study indicate that MRE11 may regulate $\mathrm{p}-\mathrm{CHK} 2$ and CDC25A expression levels under high glucose conditions. No obvious differences were observed in the expression of any other genes between groups (Fig. 4C).

The effect of high glucose on the cell viability of RWPE-1 cells was investigated and the results demonstrated that viability significantly increased with high glucose treatment (Fig. 5A and B). However, when RWPE-1 cells were transfected with cells overexpressing MRE11, cell viability decreased when incubated in high glucose solution (Fig. 5A and B). Flow cytometry revealed that cell cycle distribution was similar for normal and MRE11-overexpressing RWPE-1 cells regardless of high-glucose treatment (Fig. 5E). However, cell apoptosis analysis indicated that both the number of apoptotic normal RPEW-1 cells was increased following treatment with high-glucose solution. However, the ratios were significantly raised 2-fold when MRE11 was overexpressed and high-glucose solution was applied. Overexpression of MRE11 had no significant effect on cell apoptosis compared with normal cells without high-glucose treatment (Fig. 5D).

MRE11 expression is downregulated in diabetic prostate tissue. To measure MRE11 expression in diabetic prostate tissue, an STZ-induced diabetic rat model was established. The blood glucose concentration of rats at 2 and 4 weeks following STZ treatment were $23.07 \pm 2.86$ and $27.7 \pm 2.33 \mathrm{mM} / 1$, respectively, which indicates that the diabetes model was successfully established (Fig. 6A). The expression of MRE11 mRNA was determined by RT-qPCR. MRE11 expression was significantly downregulated in the STZ group compared with the control group (Fig. 6B). The level of proliferating cell nuclear antigen (PCNA), which is associated with cell proliferation, was also assessed. The results demonstrated that the level of PCNA in the STZ group was 2-fold higher compared with the control group (Fig. 6C). Prostate tissue samples were subjected to immunohistochemical staining, with MRE11 and PCNA stained in brown. The results of immunohistochemical staining also suggest that the expression of MRE11 

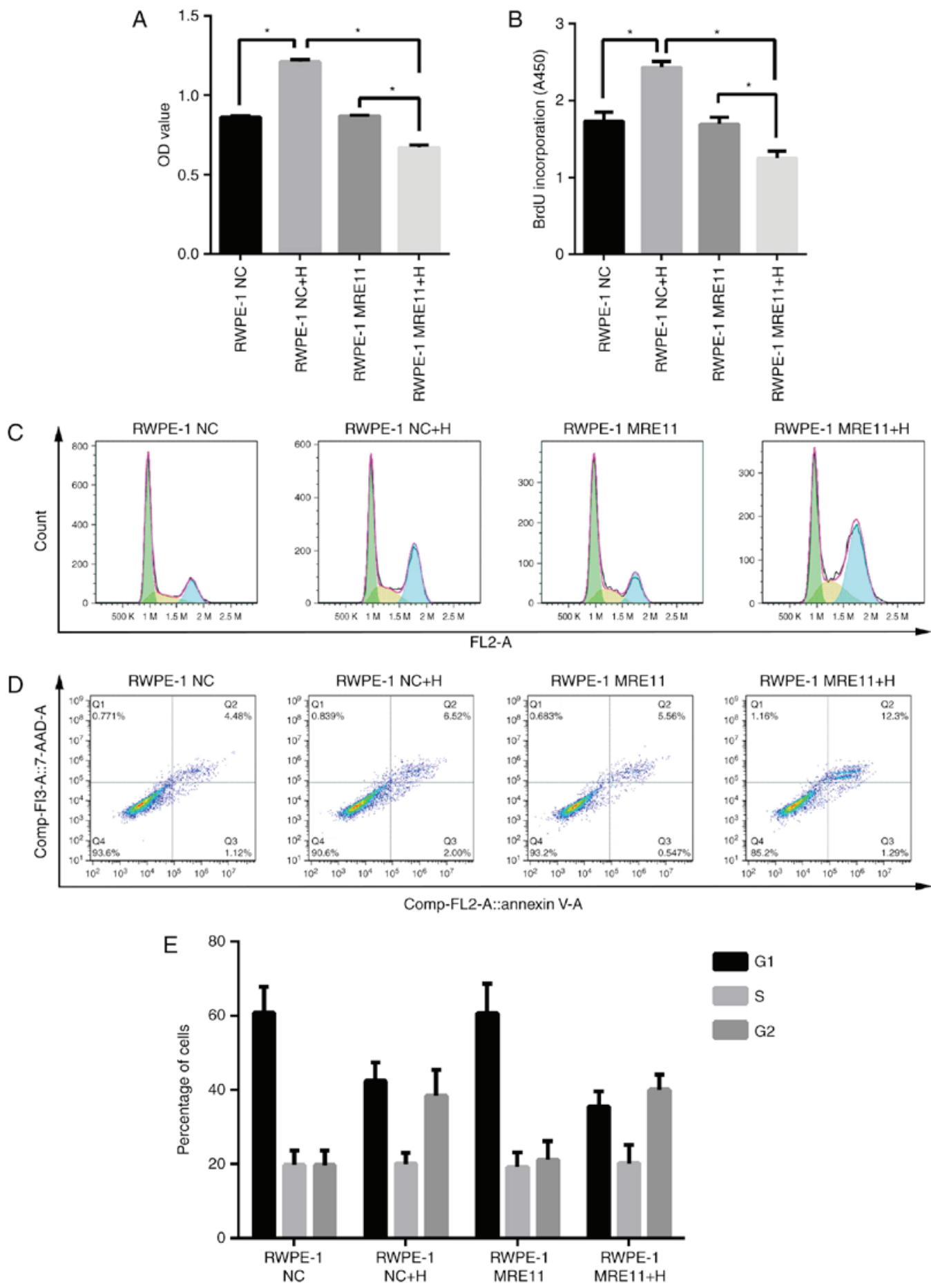

Figure 5. Cell viability was determined by (A) MTS and (B) BrdU assays. (C) Cell cycle and (D) apoptosis were analyzed by flow cytometry in RWPE-1 cells transfected with NC and MRE11-overexpression vectors with or without high glucose treatment. (E) Quantitative analysis of panel C. "P<0.05. BrdU, bromodeoxyuridine. NC, negative control; MRE11, meiotic recombination 11; H, high glucose; OD, optical density.

were decreased in the STZ group, whereas PCNA levels were increased (Fig. 6D).

\section{Discussion}

In the present study, RWPE-1 and HPr-1 cell lines were treated with a high glucose solution to investigate the effect of T2D on prostatic cell proliferation and its association with prostatic enlargement and BPH. Transcriptome sequencing indicated that MRE11 was downregulated under high glucose conditions. The overexpression of MRE11 in RWPE-1 cells revealed that the regulation of MRE11 expression is associated with cell proliferation and apoptosis in a high glucose environment. MRE11 was significantly downregulated in the prostatic tissues of rats treated with STZ.

MRE11 has been reported to activate ataxia-telangiectasia-mutated kinase 1 and induce DNA damage repair reactions $(22,23)$. The present study demonstrated that high concentrations of glucose are associated with increased intracellular oxidative stress, DNA damage, antioxidative enzymes 
A

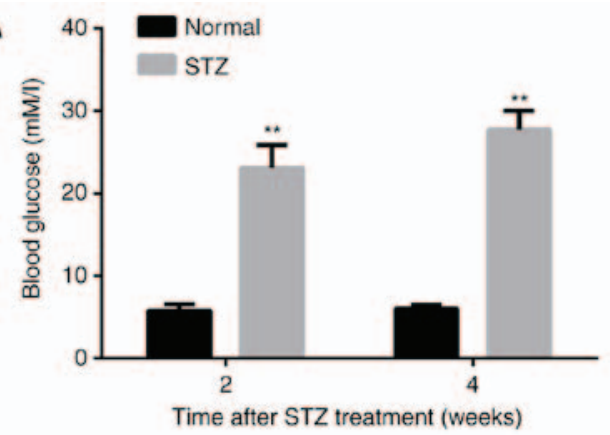

B

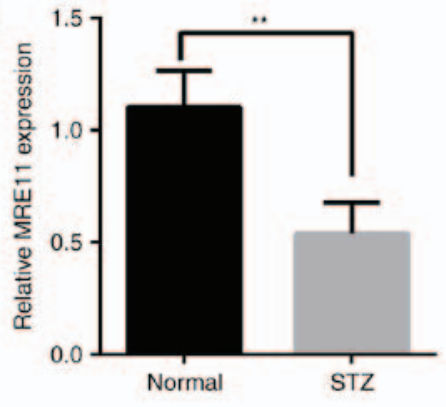

C

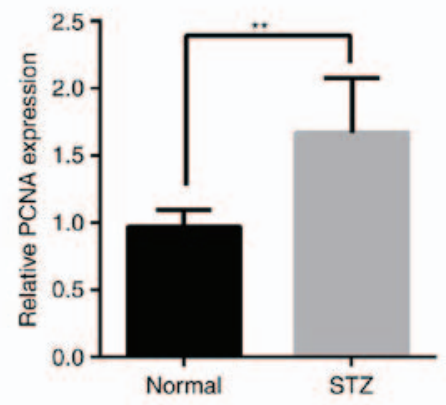

D

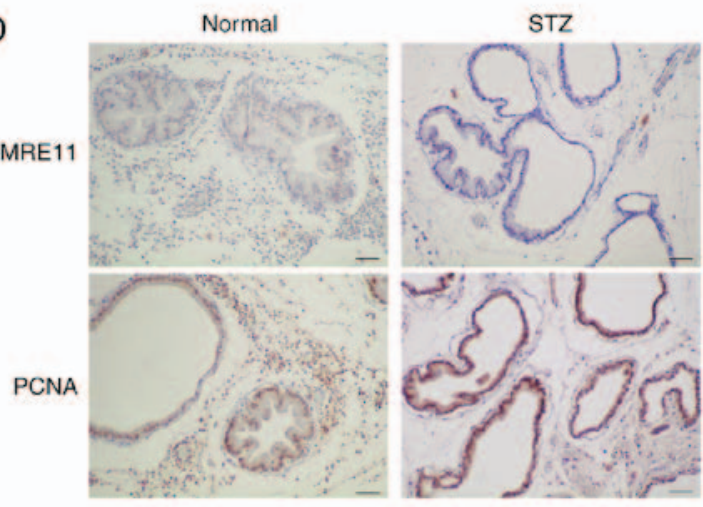

Figure 6. Expression of MRE11 and PCNA in prostatic tissues from rats with STZ-induced diabetes. (A) Blood glucose concentrations of the normal and STZ group at weeks 2 and 4 following STZ treatment. Expression of (B) MRE11 and (C) PCNA was determined by reverse transcription-quantitative polymerase chain reaction. (D) Immunochemical staining was also performed to detect the expression level of MRE11. Magnification, $\mathrm{x} 200$. Scale bar $=0.5 \mu \mathrm{m}$. $\mathrm{P}<0.01$ and ${ }^{* *} \mathrm{P}<0.01$ vs. Normal. MRE11, meiotic recombination 11; PCNA, proliferating cell nuclear antigen; STZ, Streptozotocin.

deficiency and impaired DNA damage repair machinery in the prostate. It is speculated that restoring the DNA repair machinery may protect prostate cells from oxidative DNA damage induced by high glucose (24). In the present study, MRE11 expression was downregulated under high glucose conditions, which is consistent with the results of a previous study (24). The results of the present study revealed that cell viability was significantly increased under high glucose conditions, and this increased proliferation may be an indicator of oncogenesis. MRE11 typically functions alongside other molecules, including Mre11-Rad50-Xrs2 double strand break repair complex $(25,26)$. In the present study, the expression of CHK2, CDC25A and CDK was assessed to determine whether the MRE11 overexpression vector was successfully transfected into cells. CHK2 is activated by MRE11, which in turn activates CDC25A via phosphorylation (27,28). CDK2 is an important molecule in forming the Mre11-Rad50-NBS1 (MRN) complex and is crucial to enable MRE11 to perform DNA repair functions (29). However, alterations in p-CHK2 and $\mathrm{p}-\mathrm{CDC} 25 \mathrm{~A}$ expression were observed, whereas there was no marked change in CDK2/p-CDK2 expression. Therefore, $\mathrm{CDK} / \mathrm{p}-\mathrm{CDK} 2$ does not appear to be affected by high-glucose conditions. p-CHK2 and p-CDC25A were downregulated when cells were exposed to high glucose treatment, which suggests that the DNA repair system was inhibited. p-CHK2 expression was downregulated in normal cells, whilst p-CDC25A was decreased within MRE11-overexpressing cells. This indicates that CHK2 is positively associated with MRE11 expression. It has been reported that $\mathrm{CDC} 25 \mathrm{~A}$ is regulated by $\mathrm{CHK} 2$, therefore the association between CDC25A and MRE11 may be indirect (30). According to a recent study, CHK2 deficiency in mice induces a broad spectrum of tumorigenesis (27). However, an efficient DNA repair machinery is present in normal cells to resolve DNA damage $(22,23)$; once the function of this vital machinery is compromised, cells are unable to maintain genetic stability and may undergo neoplastic transformation (27). This may explain why cell viability was increased when cells were exposed to high glucose conditions. In MRE11-overexpression cells, cell viability was decreased and cell apoptosis increased under high glucose conditions. This may be due to the toxicity of the high concentration of glucose. When MRE11 is overexpressed, the DNA repair system may be maintained in a high glucose environment; as such, differentiation into cancerous cells is impaired. The high concentration of glucose increases oxidative stress, reducing cell viability and cell apoptosis.

Results of the in vivo experiment supported the in vivo data. PCNA was used as a biomarker to analyze cell viability in prostatic tissues $(31,32)$. The results demonstrated that MRE11 expression was decreased whereas PCNA was increased. However, observations of the diabetic tissues also indicated that high glucose conditions induced cell enlargement, a common indicator of BPH. These results suggest that diabetes may induce BPH. MRE11 also serves an important role during the pathogenesis of $\mathrm{BPH}$ and may be considered as a biomarker for the diagnosis of $\mathrm{BPH}$ or diabetes, as well as a novel therapeutic target.

The results of the present study indicate that there may be an association between BPH and diabetes. MRE11 expression was downregulated under high glucose conditions and in in a rat model of diabetes. To further validate the importance of MRE11, further studies utilizing other diabetic animal models 
should be performed. Effective clinical assays to measure the expression of MRE11 are also required to accurately diagnose and develop effective treatments for of BPH and diabetes.

\section{Acknowledgements}

The present study was supported by the Yunnan Applied Basic Research Project (grant no. 2014FB058) and the National Natural Science Foundation of China (grant nos. 81560417 and 81460385).

\section{Competing interests}

The authors declare that they have no competing interests.

\section{References}

1. Gandhi J, Weissbart SJ, Smith NL, Kaplan SA, Dagur G, Zumbo A, Joshi G and Khan SA: The impact and management of sexual dysfunction secondary to pharmacological therapy of benign prostatic hyperplasia. Transl Androl Urol 6: 295-304, 2017.

2. Komninos C and Mitsogiannis I: Obstruction-induced alterations within the urinary bladder and their role in the pathophysiology of lower urinary tract symptomatology. Can Urol Assoc J 8: E524-E530, 2014.

3. Schwartz I, Wein AJ, Malloy TR and Glick JH: Prostatic cancer after prostatectomy for benign disease. Cancer 58: 994-996, 1986.

4. Hua L, Zhang J, Wu H, Sui Y, Zhang W, Qian L and Wang Z: Prostate cancer after prostatectomy for benign prostatic hyperplasia. Zhonghua Nan Ke Xue 10: 612-613, 2004 (In Chinese).

5. Kristal AR, Arnold KB, Schenk JM, Neuhouser ML, Weiss N, Goodman P, Antvelink CM, Penson DF and Thompson IM: Race/ethnicity, obesity, health related behaviors and the risk of symptomatic benign prostatic hyperplasia: Results from the prostate cancer prevention trial. J Urol 177: 1395-1400, 2007.

6. Qu X, Huang Z, Meng X, Zhang X, Dong L and Zhao X: Prostate volume correlates with diabetes in elderly benign prostatic hyperplasia patients. Int Urol Nephrol 46: 499-504, 2014.

7. Chen Z, Miao L, Gao X, Wang G and Xu Y: Effect of obesity and hyperglycemia on benign prostatic hyperplasia in elderly patients with newly diagnosed type 2 diabetes. Int J Clin Exp Med 8: 11289-11294, 2015.

8. Kahn SE, Cooper ME and Del Prato S: Pathophysiology and treatment of type 2 diabetes: Perspectives on the past, present, and future. Lancet 383: 1068-1083, 2014.

9. Raccah D: Insulin therapy in patients with type 2 diabetes mellitus: Treatment to target fasting and postprandial blood glucose levels. Insulin 1: 158-165, 2006.

10. Breyer BN and Sarma AV: Hyperglycemia and insulin resistance and the risk of BPH/LUTS: An update of recent literature. Curr Urol Rep 15: 462, 2014.

11. Fleshner NE and Bhindi B: Metabolic syndrome and diabetes for the urologist. Can Urol Assoc J 8 (7-8 Suppl 5): S159-S161, 2014.

12. Shimizu S, Tsounapi P, Shimizu T, Honda M, Inoue K, Dimitriadis F and Saito M: Lower urinary tract symptoms, benign prostatic hyperplasia/benign prostatic enlargement and erectile dysfunction: Are these conditions related to vascular dysfunction? Int J Urol 21: 856-864, 2014.

13. Majewski N, Nogueira V, Robey RB and Hay N: Akt inhibits apoptosis downstream of BID cleavage via a glucose-dependent mechanism involving mitochondrial hexokinases. Mol Cell Biol 24: 730-740, 2004

14. Gottlob K, Majewski N, Kennedy S, Kandel E, Robey RB and Hay N: Inhibition of early apoptotic events by Akt/PKB is dependent on the first committed step of glycolysis and mitochondrial hexokinase. Genes Dev 15: 1406-1418, 2001.
15. Medema RH, Kops GJ, Bos JL and Burgering BM: AFX-like Forkhead transcription factors mediate cell-cycle regulation by Ras and PKB through p27kip1. Nature 404: 782-787, 2000.

16. Buteau J and Accili D: Regulation of pancreatic beta-cell function by the forkhead protein FoxO1. Diabetes Obes Metab 9 (Suppl 2): S140-S146, 2007.

17. Glauser DA and Schlegel W: The emerging role of FOXO transcription factors in pancreatic beta cells. J Endocrinol 193: 195-207, 2007.

18. Chunwei YX, Yu W, Baoyi Z, Ya W, Shujun F, Zebin C and Xingqiao W: Morphological and genetic changes in the prostate of rats with diabetes. Chin J Exp Surg 28: 1535-1538, 2011.

19. Trapnell C, Pachter L and Salzberg SL: TopHat: Discovering splice junctions with RNA-Seq. Bioinformatics 25: 1105-1111, 2009.

20. Benjamini Y and Hochberg Y: Controlling the false discovery rate-a practical and powerful approach to multiple testing. J R Statistical Soc 57: 289-300, 1995.

21. Livak KJ and Schmittgen TD: Analysis of relative gene expression data using real-time quantitative PCR and the 2(-Delta Delta C(T)) method. Methods 25: 402-408, 2001.

22. Czornak K, Chughtai S and Chrzanowska KH: Mystery of DNA repair: The role of the MRN complex and ATM kinase in DNA damage repair. J Appl Genet 49: 383-396, 2008.

23. Zha S, Boboila $C$ and Alt FW: Mre11: Roles in DNA repair beyond homologous recombination. Nat Struct Mol Biol 16: 798-800, 2009.

24. Ye C, Li X, Wang Y, Zhang Y, Cai M, Zhu B, Mu P, Xia X, Zhao Y, Weng J, et al: Diabetes causes multiple genetic alterations and downregulates expression of DNA repair genes in the prostate. Lab Invest 91: 1363-1374, 2011.

25. Paull TT and Gellert M: A mechanistic basis for Mre11-directed DNA joining at microhomologies. Proc Natl Acad Sci USA 97: 6409-6414, 2000.

26. Zhang $X$ and Paull TT: The Mre11/Rad50/Xrs2 complex and non-homologous end-joining of incompatible ends in S. cerevisiae. DNA Repair (Amst) 4: 1281-1294, 2005.

27. Inagaki A, Roset R and Petrini JH: Functions of the MRE11 complex in the development and maintenance of oocytes. Chromosoma 125: 151-162, 2016.

28. Zhao H, Traganos F, Albino AP and Darzynkiewicz Z: Oxidative stress induces cell cycle-dependent Mre11 recruitment, ATM and Chk2 activation and histone $\mathrm{H} 2 \mathrm{AX}$ phosphorylation. Cell Cycle 7: 1490-1495, 2008.

29. Buis J, Stoneham T, Spehalski E and Ferguson DO: Mre11 regulates CtIP-dependent double-strand break repair by interaction with CDK2. Nat Struct Mol Biol 19: 246-252, 2012.

30. Myer DL, Robbins SB, Yin M, Boivin GP, Liu Y, Greis KD, Bahassi el $\mathrm{M}$ and Stambrook PJ: Absence of polo-like kinase 3 in mice stabilizes Cdc25A after DNA damage but is not sufficient to produce tumors. Mutat Res 714: 1-10, 2011.

31. Bryja A, Dyszkiewicz-Konwinska M, Budna J, Ciesiółka S, Kranc W, Borys S, Jeseta M, Urbaniak O, Bukowska D, Antosik P, et al: Expression of cell mitotic progression proteins and keratinocyte markers in porcine buccal pouch mucosal cells during short-term, real-time primary culture. J Biol Regul Homeost Agents 31: 297-309, 2017.

32. Sales CF, Santos KPED, Rizzo E, Ribeiro RIMA, Santos HBD and Thomé RG: Proliferation, survival and cell death in fish gills remodeling: From injury to recovery. Fish Shellfish Immunol 68: 10-18, 2017.

This work is licensed under a Creative Commons Attribution-NonCommercial-NoDerivatives 4.0 International (CC BY-NC-ND 4.0) License. 